\title{
Dual Theory for Matrix Linear Bilevel Programming
}

\author{
Yun Wang ${ }^{*}$, Hao Xu \\ College of Information Science and Engineering, Shandong Agricultural University, Taian, China.
}

How to cite this paper: Yun Wang, Hao Xu. (2020) Dual Theory for Matrix Linear Bilevel Programming. Journal of Applied Mathematics and Computation, 4(4), 147-152.

DOI:10.26855/jamc.2020.12.006

Received: September 15, 2020

Accepted: October 12, 2020

Published: November 6, 2020

*Corresponding author: Yun Wang, College of Information Science and Engineering, Shandong Agricultural University, Taian, China.

Email: wangyun@sdau.edu.cn

\begin{abstract}
Based on the research of the linear bilevel programming, a matrix linear bilevel programming is considered in this paper. Firstly, the equivalent single-level programming form is given. Then, the Lagrange duality theory is used to discuss the duality programming problems and properties of matrix-type linear bilevel programming. Finally, the relationship between the saddle condition of the duality linear programming and optimal solution of the matrix linear bilevel programming is discussed.
\end{abstract}

\section{Keywords}

Matrix Linear Bilevel Programming, Dual Theory, Lagrange Duality Programming, Saddle Condition

\section{Introduction}

Bilevel programming was proposed by Bracken and McGill in a series of literatures [1] in the 1970s as the following form:

$$
\begin{array}{ll}
\min & f(x, y) \\
\text { s.t } & g(x, y) \geq 0 \\
& h(x, y)=0 \\
& y=\arg \min \{F(x, y) \mid G(x, y) \geq 0, H(x, y)=0\}
\end{array}
$$

where, $f: R^{n} \rightarrow R, g: R^{n} \rightarrow R^{m}, h: R^{n} \rightarrow R, F: R^{n} \rightarrow R, G: R^{n} \rightarrow R^{p}, H: R^{n} \rightarrow R^{q}$.

With the development of science and technology, the application of bilevel programming in transportation, management decision-making, energy policy, engineering design and so on is becoming more and more extensive [2,3]. Meanwhile, the form of bilevel programming was expanded. The optimization was no longer limited to one parameter constraint, but contained variable inequalities or complementary constraints [4, 5]. In 1988, Harker and Pang [6] introduced different professional terms namely, mathematical programming with equilibrium constraints, for short, MPEC. In the study of the optimal conditions of the bilevel programming problem, the most common method is to replace the lower-level problem with its optimal conditions to obtain a single-level programming problem. The resulting single-level programming problem is the MPEC. The detailed concepts and relevant theories of MPEC are given in [6, 7].

Optimality conditions can be used to characterize the properties of optimal solution and guarantee the convergence of algorithm. The dual theory is a very important part in the study of the optimal conditions. Romano-Rodriquez established the first dual theory of bilevel programming in 1994 [5]. In recent years, there has been a lot of research on the dual problem of linear bilevel programming. But the study of the dual theory is limited to vector space and lacks matrix space [8]. In this paper, it is focused on the optimal conditions of matrix-type linear bilevel programming, by using the dual theory and 
saddle condition.

The structure of this paper is as follows. In Section 2, we introduce some preliminaries about bilevel programming and semidefinite programming. The Lagrange duality programming is given in section 3 . In the last section, the relationship between saddle point and optimal solution is studied.

\section{Preliminaries}

In this section, we will introduce some basic knowledge about bilevel programming [9, 10].

Considering the following problem

$$
\begin{aligned}
& \min \left\langle C_{1}, X\right\rangle+\left\langle D_{1}, Y\right\rangle \\
& \left\{\begin{array}{l}
\mathcal{A}_{1} X+\mathcal{B}_{1} Y \geq b_{1} \\
Y \in \operatorname{argmin}\left\{\left\langle C_{2}, X\right\rangle+\left\langle D_{2}, Y\right\rangle: \mathcal{A}_{2} X+\mathcal{B}_{2} Y \geq b_{2}\right\} \\
X \in S_{+}^{n}, Y \in S_{+}^{n}
\end{array},\right.
\end{aligned}
$$

where $X \in S^{n}, Y \in S^{n}$ are symmetric matrices, $C_{i}, D_{i} \in S^{n}, b_{i} \in R^{n}, \mathcal{A}_{i}, \mathcal{B}_{i}: S^{n} \rightarrow R^{m}, i=1,2$ are linear transformations, Obviously, (2.1) has the same solution as the following programming:

$$
\begin{aligned}
& \min \left\langle C_{1}, X\right\rangle+\left\langle D_{1}, Y\right\rangle \\
& \left\{\begin{array}{l}
\mathcal{A}_{1} X+\mathcal{B}_{1} Y \geq b_{1} \\
Y \in \operatorname{argmin}\left\{\left\langle D_{2}, Y\right\rangle: \mathcal{A}_{2} X+\mathcal{B}_{2} Y \geq b_{2}\right\} \\
X \in S_{+}^{n}, Y \in S_{+}^{n}
\end{array} .\right.
\end{aligned}
$$

According to the treatment method of linear bilevel programming, it is easy to draw the following conclusions [9].

Lemma 2.1 The above programming (2.2) is equivalent to the following one:

$$
\begin{aligned}
& \min \left\langle C_{1}, X\right\rangle+\left\langle D_{1}, Y\right\rangle \\
& \text { s.t }\left\{\begin{array}{l}
b_{1}-\mathcal{A}_{1} X-\mathcal{B}_{1} Y \leq 0 \\
b_{2}-\mathcal{A}_{2} X-\mathcal{B}_{2} Y \leq 0 \\
\mathcal{B}_{2}^{*} \lambda-D_{2} \preceq 0 \\
\left\langle\mathcal{A}_{2} X+\mathcal{B}_{2} Y-b_{2}, \lambda\right\rangle=0 \\
\left\langle D_{2}-\mathcal{B}_{2}^{*} \lambda, Y\right\rangle=0 \\
X, Y, \lambda \succeq 0
\end{array},\right.
\end{aligned}
$$

where $\lambda \in S^{n}, \mathcal{B}^{*}$ is the conjugate of $\mathcal{B}$, satisfying $\langle\mathcal{B} Y, \lambda\rangle=\left\langle Y, \mathcal{B}^{*} \lambda\right\rangle$.

We denote the definition of feasible domain, feasible solution set and optimal solution set of matrix linear bilevel programming as follows:

1) The feasible domain for the original question (2.2) is denoted by

$$
P=\left\{(X, Y) \mid \mathcal{A}_{1} X+\mathcal{B}_{1} Y \geq b_{1}, \mathcal{A}_{2} X+\mathcal{B}_{2} Y \geq b_{2}, X \succeq 0, Y \succeq 0\right\} ;
$$

2) For any $X \in S^{n}$, the feasible solution set for the lower-level programming is

$$
P(X)=\left\{Y \in S^{n} \mid \mathcal{A}_{2} X+\mathcal{B}_{2} Y \geq b_{2}, Y \succeq 0\right\} ;
$$

3) Let $\bar{P}=\left\{(X, Y) \in P \mid Y \in \arg \min _{Y \in P(X)}\left\langle D_{2}, Y\right\rangle\right\}$ be the feasible solution set. 


\section{Lagrange duality}

This section mainly uses the Lagrange dual of the equivalent single-level problem $(P)$ to construct the Lagrange duality of the matrix linear bilevel programming. Then the basic dual theories such as the properties of weak and strong duality are proved.

Let

then

$$
\begin{aligned}
\varphi(\gamma, \mu)= & \inf _{Z, Y, \lambda \succeq 0}\left\{\left\langle C_{1}, X\right\rangle+\left\langle D_{1}, Y\right\rangle+\left\langle\gamma_{1}, b_{1}-\mathcal{A}_{1} X-\mathcal{B}_{1} Y\right\rangle+\left\langle\gamma_{2}, b_{2}-\mathcal{A}_{2} X-\mathcal{B}_{2} Y\right\rangle\right. \\
& +\left\langle\gamma_{3}, \mathcal{B}_{2}^{*} \lambda-D_{2}\right\rangle+\mu_{1}\left[\left\langle\mathcal{A}_{2} X+\mathcal{B}_{2} Y-b_{2}, \lambda\right\rangle\right]+\mu_{2}\left[\left\langle D_{2}-\mathcal{B}_{2}^{*} \lambda, Y\right\rangle\right],
\end{aligned}
$$

$\max \varphi(\gamma, \mu)$

(D) S.t $\gamma_{1}, \gamma_{1} \geq 0, \quad \gamma_{3} \pm 0$ '

is the dual of (3.2). Denoted $D$ to be the feasible domain of $(D)$.

Theorem 3.1 Let $(\bar{X}, \bar{Y}, \bar{\lambda})$ be the feasible solution to the original problem. $(P),(\gamma, \mu)$ be the feasible solution to dual problem $(D)$. Then

$$
\left\langle C_{1}, X\right\rangle+\left\langle D_{1}, Y\right\rangle \geq \varphi(\gamma, \mu),
$$

i.e., the dual gap is nonnegative at any feasible solution.

Proof: As $(\bar{X}, \bar{Y}, \bar{\lambda}) \in P$,

$$
\left\{\begin{array}{l}
b_{1}-\mathcal{A}_{1} \bar{X}-\mathcal{B}_{1} \bar{Y} \leq 0 \\
b_{2}-\mathcal{A}_{2} \bar{X}-\mathcal{B}_{2} \bar{Y} \leq 0 \\
\mathcal{B}_{2}{ }^{*} \bar{\lambda}-D_{2} \prec 0 \\
\left\langle\mathcal{A}_{2} \bar{X}+\mathcal{B}_{2} \bar{Y}-b_{2}, \bar{\lambda}\right\rangle=0 \\
\left\langle D_{2}-\mathcal{B}_{2}{ }^{*} \bar{\lambda}, \bar{Y}\right\rangle=0
\end{array}\right.
$$

and $\gamma_{1}, \gamma_{2} \geq 0, \gamma_{3} \succeq 0$. Therefore, from the property of dual programming, we can get the conclusion:

$$
\begin{aligned}
\varphi(\gamma, \mu)= & \inf _{Z, Y, \lambda \geq 0}\left\{\left\langle C_{1}, X\right\rangle+\left\langle D_{1}, Y\right\rangle+\left\langle\gamma_{1}, b_{1}-\mathcal{A}_{1} X-\mathcal{B}_{1} Y\right\rangle+\left\langle\gamma_{2}, b_{2}-\mathcal{A}_{2} X-\mathcal{B}_{2} Y\right\rangle\right. \\
& +\left\langle\gamma_{3}, \mathcal{B}_{2}^{*} \lambda-D_{2}\right\rangle+\mu_{1}\left[\left\langle\mathcal{A}_{2} X+\mathcal{B}_{2} Y-b_{2}, \lambda\right\rangle\right]+\mu_{2}\left[\left\langle D_{2}-\mathcal{B}_{2}^{*} \lambda, Y\right\rangle\right] \\
\leq & \left\langle C_{1}, \bar{X}\right\rangle+\left\langle D_{1}, \bar{Y}\right\rangle+\left\langle\gamma_{1}, b_{1}-\mathcal{A}_{1} \bar{X}-\mathcal{B}_{1} \bar{Y}\right\rangle+\left\langle\gamma_{2}, b_{2}-\mathcal{A}_{2} \bar{X}-\mathcal{B}_{2} \bar{Y}\right\rangle \\
& +\left\langle\gamma_{3}, \mathcal{B}_{2}^{*} \bar{\lambda}-D_{2}\right\rangle+\mu_{1}\left[\left\langle\mathcal{A}_{2} \bar{X}+\mathcal{B}_{2} \bar{Y}-b_{2}, \bar{\lambda}\right\rangle\right]+\mu_{2}\left[\left\langle D_{2}-\mathcal{B}_{2}^{*} \bar{\lambda}, \bar{Y}\right\rangle\right] \\
\leq & \left\langle C_{1}, \bar{X}\right\rangle+\left\langle D_{1}, \bar{Y}\right\rangle .
\end{aligned}
$$

From theorem 3.1, we can get the following three corollaries:

Corollary 3.1 In questions $(P)$ and $(D)$,

$$
\inf \left\{\left\langle C_{1}, X\right\rangle+\left\langle D_{1}, Y\right\rangle \mid X, Y \in \bar{P}\right\} \geq \sup \left\{\varphi(\gamma, \mu) \mid \gamma_{1}, \gamma_{2} \geq 0, \gamma_{3} \succeq 0\right\} .
$$

Corollary 3.2 If $\left\langle C_{1}, \bar{X}\right\rangle+\left\langle D_{1}, \bar{Y}\right\rangle \leq \varphi(\bar{\gamma}, \bar{\mu}), \bar{\gamma}_{1}, \bar{\gamma}_{2} \geq 0, \bar{\gamma}_{3} \succeq 0$, and $(\bar{X}, \bar{Y}, \bar{\lambda})$ is the feasible solution to the original problem. Then $(\bar{X}, \bar{Y}, \bar{\lambda})$ is the minimum point of $(P),(\bar{\gamma}, \bar{\mu})$ is the maximum point of $(D)$.

Corollary 3.3

(i) If $\inf _{X, Y, \lambda \succeq 0}\left\{\left\langle C_{1}, X\right\rangle+\left\langle D_{1}, Y\right\rangle\right\}=-\infty$, then $\forall \gamma_{1}, \gamma_{2} \geq 0, \gamma_{3} \succeq 0, \varphi(\gamma, \mu)=-\infty$;

(ii) If $\sup _{\gamma_{1}, \gamma_{2} \geq 0, \gamma_{3} \succeq 0}\{\varphi(\gamma, \mu)\}=\infty$, then $(P)$ has no feasible solution, i.e., $\bar{P}=\varnothing$. 
Theorem 3.2 (strong duality) If both problems $(P)$ and $(D)$ are feasible and Slater regularity condition holds, then the optimal solution sets of problems $(P)$ and $(D)$ are not empty, and the optimal values of the two problems are equal. It implies that if there exists $(X, Y, \lambda) \in \bar{P}$, such that

$$
\left\{\begin{array}{l}
b_{1}-\mathcal{A}_{1} X-\mathcal{B}_{1} Y<0 \\
b_{2}-\mathcal{A}_{2} X-\mathcal{B}_{2} Y<0 \\
\mathcal{B}_{2}{ }^{*} \lambda-D_{2} \prec 0 \\
\left\langle\mathcal{A}_{2} X+\mathcal{B}_{2} Y-b_{2}, \lambda\right\rangle<0 \\
\left\langle D_{2}-\mathcal{B}_{2}{ }^{*} \lambda, Y\right\rangle<0
\end{array} .\right.
$$

Then

$$
\inf _{X, Y, \lambda \succeq 0}\left\{\left\langle C_{1}, X\right\rangle+\left\langle D_{1}, Y\right\rangle\right\}=\sup _{\gamma_{1}, \gamma_{2} \geq 0, \gamma_{3} \succeq 0}\{\varphi(\gamma, \mu)\} .
$$

Proof: It can be seen from the feasibility of the problems, there exists $(\bar{X}, \bar{Y}) \in P$ and $(\bar{\gamma}, \bar{\mu}) \in D$, such that

$$
\begin{aligned}
& p^{*}=\inf _{X, Y \in \bar{P}}\left\langle C_{1}, X\right\rangle+\left\langle D_{1}, Y\right\rangle \geq \varphi(\bar{\gamma}, \bar{\mu})>-\infty ; \\
& d^{*}=\sup _{\gamma, \mu \in D} \varphi(\gamma, \mu) \leq\left\langle C_{1}, \bar{X}\right\rangle+\left\langle D_{1}, \bar{Y}\right\rangle<+\infty,
\end{aligned}
$$

which implies the optimal solution sets of problems $(P)$ and $(D)$ are not empty. Combining with the Slater condition, it obtains $p^{*}=d^{*}$.

\section{Saddle condition and optimal solution}

In order to discuss the relationship between the optimality condition of matrix linear bilevel programming and the saddle point of Lagrange function, we first give the concept of saddle point condition and related theorems, and then discuss the relationship between saddle point and optimal point.

Considering programming $(P)$, if $\bar{X}, \bar{Y}, \bar{\lambda} \succeq 0, \bar{\gamma}_{1}, \bar{\gamma}_{2} \geq 0, \bar{\gamma}_{3} \succeq 0, \bar{\mu}>0$, such that Lagrange function

$$
\begin{aligned}
L(X, Y, \lambda, \gamma, \mu)= & \left\langle C_{1}, X\right\rangle+\left\langle D_{1}, Y\right\rangle+\left\langle\gamma_{1}, b_{1}-\mathcal{A}_{1} X-\mathcal{B}_{1} Y\right\rangle+\left\langle\gamma_{2}, b_{2}-\mathcal{A}_{2} X-\mathcal{B}_{2} Y\right\rangle+\left\langle\gamma_{3}, \mathcal{B}_{2}{ }^{*} \lambda-D_{2}\right\rangle \\
& +\mu_{1}\left[\left\langle\mathcal{A}_{2} X+\mathcal{B}_{2} Y-b_{2}, \lambda\right\rangle\right]+\mu_{2}\left[\left\langle D_{2}-\mathcal{B}_{2}{ }^{*} \lambda, Y\right\rangle\right]
\end{aligned}
$$

holds

$$
L(\bar{X}, \bar{Y}, \bar{\lambda}, \gamma, \mu) \leq L(\bar{X}, \bar{Y}, \bar{\lambda}, \bar{\gamma}, \bar{\mu}) \leq L(X, Y, \lambda, \bar{\gamma}, \bar{\mu}),
$$

then $(\bar{X}, \bar{Y}, \bar{\lambda}, \bar{\gamma}, \bar{\mu})$ is called the saddle point of the Lagrange function.

Theorem 4.1 The following functions $P(X, Y, \lambda)=\left\langle\mathcal{A}_{2} X+\mathcal{B}_{2} Y-b_{2}, \lambda\right\rangle$ and $Q(\lambda, Y)=\left\langle D_{2}-\mathcal{B}_{2}^{*} \lambda, Y\right\rangle$ are convex.

Proof: It is easy to verify with definitions of convexity that $P(X, Y, \lambda)$ and $Q(\lambda, Y)$ are convex.

Theorem 4.2 If $(\bar{X}, \bar{Y}, \bar{\lambda}, \bar{\gamma}, \bar{\mu})$ is the saddle point of the Lagrange function, then $(\bar{X}, \bar{Y}, \bar{\lambda})$ and $(\bar{\gamma}, \bar{\mu})$ are the solutions of the two problems $(P)$ and $(D)$, respectively.

Proof: Let $(\bar{X}, \bar{Y}, \bar{\lambda}, \bar{\gamma}, \bar{\mu})$ be the saddle point of the Lagrange function, then 


$$
\begin{aligned}
& \left\langle C_{1}, \bar{X}\right\rangle+\left\langle D_{1}, \bar{Y}\right\rangle+\left\langle\gamma_{1}, b_{1}-\mathcal{A}_{1} \bar{X}-\mathcal{B}_{1} \bar{Y}\right\rangle+\left\langle\gamma_{2}, b_{2}-\mathcal{A}_{2} \bar{X}-\mathcal{B}_{2} \bar{Y}\right\rangle+\left\langle\gamma_{3}, \mathcal{B}_{2}^{*} \bar{\lambda}-D_{2}\right\rangle \\
& +\mu_{1}\left[\left\langle\mathcal{A}_{2} \bar{X}+\mathcal{B}_{2} \bar{Y}-b_{2}, \bar{\lambda}\right\rangle\right]+\mu_{2}\left[\left\langle D_{2}-\mathcal{B}_{2}^{*} \bar{\lambda}, \bar{Y}\right\rangle\right] \\
\leq & \left\langle C_{1}, \bar{X}\right\rangle+\left\langle D_{1}, \bar{Y}\right\rangle+\left\langle\bar{\gamma}_{1}, b_{1}-\mathcal{A}_{1} \bar{X}-\mathcal{B}_{1} \bar{Y}\right\rangle+\left\langle\bar{\gamma}_{2}, b_{2}-\mathcal{A}_{2} \bar{X}-\mathcal{B}_{2} \bar{Y}\right\rangle+\left\langle\bar{\gamma}_{3}, \mathcal{B}_{2}^{*} \bar{\lambda}-D_{2}\right\rangle \\
& +\bar{\mu}_{1}\left[\left\langle\mathcal{A}_{2} \bar{X}+\mathcal{B}_{2} \bar{Y}-b_{2}, \bar{\lambda}\right\rangle\right]+\bar{\mu}_{2}\left[\left\langle D_{2}-\mathcal{B}_{2}^{*} \bar{\lambda}, \bar{Y}\right\rangle\right] \\
\leq & \left\langle C_{1}, X\right\rangle+\left\langle D_{1}, Y\right\rangle+\left\langle\bar{\gamma}_{1}, b_{1}-\mathcal{A}_{1} X-\mathcal{B}_{1} Y\right\rangle+\left\langle\bar{\gamma}_{2}, b_{2}-\mathcal{A}_{2} X-\mathcal{B}_{2} Y\right\rangle+\left\langle\bar{\gamma}_{3}, \mathcal{B}_{2}^{*} \lambda-D_{2}\right\rangle \\
& +\bar{\mu}_{1}\left[\left\langle\mathcal{A}_{2} X+\mathcal{B}_{2} Y-b_{2}, \lambda\right\rangle\right]+\bar{\mu}_{2}\left[\left\langle D_{2}-\mathcal{B}_{2}^{*} \lambda, Y\right\rangle\right] .
\end{aligned}
$$

For the first inequality (4.1),

$$
\begin{aligned}
& \left\langle\gamma_{1}-\bar{\gamma}_{1}, b_{1}-\mathcal{A}_{1} \bar{X}-\mathcal{B}_{1} \bar{Y}\right\rangle+\left\langle\gamma_{2}-\bar{\gamma}_{2}, b_{2}-\mathcal{A}_{2} \bar{X}-\mathcal{B}_{2} \bar{Y}\right\rangle+\left\langle\gamma_{3}-\bar{\gamma}_{3}, \mathcal{B}_{2}^{*} \bar{\lambda}-D_{2}\right\rangle \\
& +\left(\mu_{1}-\bar{\mu}_{1}\right)\left[\left\langle\mathcal{A}_{2} \bar{X}+\mathcal{B}_{2} \bar{Y}-b_{2}, \bar{\lambda}\right\rangle\right]+\left(\mu_{2}-\bar{\mu}_{2}\right)\left[\left\langle D_{2}-\mathcal{B}_{2}{ }^{*} \lambda, \bar{Y}\right\rangle\right] \leq 0 .
\end{aligned}
$$

Next we prove $\left\langle\mathcal{A}_{2} X+\mathcal{B}_{2} Y-b_{2}, \lambda\right\rangle=0$ and $\left\langle D_{2}-\mathcal{B}_{2}^{*} \lambda, Y\right\rangle=0$ :

Assume that $\left\langle\mathcal{A}_{2} X+\mathcal{B}_{2} Y-b_{2}, \lambda\right\rangle>0$, let $\gamma_{1}=\bar{\gamma}_{1}, \gamma_{2}=\bar{\gamma}_{2}, \gamma_{3}=\bar{\gamma}_{3}, \mu_{2}=\bar{\mu}_{2}, \mu_{1}=\bar{\mu}_{1}+1$, then it gets:

$$
\begin{aligned}
& \left\langle\gamma_{1}-\bar{\gamma}_{1}, b_{1}-\mathcal{A}_{1} \bar{X}-\mathcal{B}_{1} \bar{Y}\right\rangle+\left\langle\gamma_{2}-\bar{\gamma}_{2}, b_{2}-\mathcal{A}_{2} \bar{X}-\mathcal{B}_{2} \bar{Y}\right\rangle+\left\langle\gamma_{3}-\bar{\gamma}_{3}, \mathcal{B}_{2}^{*} \bar{\lambda}-D_{2}\right\rangle \\
& +\left(\mu_{1}-\bar{\mu}_{1}\right)\left[\left\langle\mathcal{A}_{2} \bar{X}+\mathcal{B}_{2} \bar{Y}-b_{2}, \bar{\lambda}\right\rangle\right]+\left(\mu_{2}-\bar{\mu}_{2}\right)\left[\left\langle D_{2}-\mathcal{B}_{2}^{*} \lambda, \bar{Y}\right\rangle\right]>0 .
\end{aligned}
$$

which is in contradiction with the result of (4.2). Similarly, $\left\langle\mathcal{A}_{2} X+\mathcal{B}_{2} Y-b_{2}, \lambda\right\rangle<0$ derives contradiction, thus $\left\langle\mathcal{A}_{2} X+\mathcal{B}_{2} Y-b_{2}, \lambda\right\rangle=0$.

The same can be proved that $\left\langle D_{2}-\mathcal{B}_{2}{ }^{*} \lambda, Y\right\rangle=0$, then $(\bar{X}, \bar{Y}, \bar{\lambda})$ is the feasible solutions of $(P)$.

Let $\gamma_{1}=\bar{\gamma}_{1}+1, \gamma_{2}=\bar{\gamma}_{2}, \gamma_{3}=\bar{\gamma}_{3}, \mu_{2}=\bar{\mu}_{2}, \mu_{1}=\bar{\mu}_{1}$, it follows from (4.1) that

$$
\mathcal{A}_{1} X+\mathcal{B}_{1} Y-b_{1} \leq 0, \quad \mathcal{A}_{2} X+\mathcal{B}_{2} Y-b_{2} \leq 0 \quad \mathcal{B}_{2}^{*} \lambda-D_{2} \preceq 0 .
$$

If $\gamma=0$,

$$
-\left\langle\bar{\gamma}_{1}, b_{1}-\mathcal{A}_{1} \bar{X}-\mathcal{B}_{1} \bar{Y}\right\rangle-\left\langle\bar{\gamma}_{2}, b_{2}-\mathcal{A}_{2} \bar{X}-\mathcal{B}_{2} \bar{Y}\right\rangle-\left\langle\bar{\gamma}_{3}, \mathcal{B}_{2}{ }^{*} \bar{\lambda}-D_{2}\right\rangle \leq 0 .
$$

but

$$
\gamma=0, \quad b_{1}-\mathcal{A}_{1} X-\mathcal{B}_{1} Y \leq 0, \quad b_{2}-\mathcal{A}_{2} X-\mathcal{B}_{2} Y \leq 0, \quad \mathcal{B}_{2}{ }^{*} \lambda-D_{2} \preceq 0,
$$

Therefore,

$$
\left\langle\bar{\gamma}_{1}, b_{1}-\mathcal{A}_{1} \bar{X}-\mathcal{B}_{1} \bar{Y}\right\rangle+\left\langle\bar{\gamma}_{2}, b_{2}-\mathcal{A}_{2} \bar{X}-\mathcal{B}_{2} \bar{Y}\right\rangle+\left\langle\bar{\gamma}_{3}, \mathcal{B}_{2}{ }^{*} \bar{\lambda}-D_{2}\right\rangle=0 .
$$

Furthermore,

$$
\begin{aligned}
\left\langle C_{1}, \bar{X}\right\rangle+\left\langle D_{1}, \bar{Y}\right\rangle \leq & \left\langle C_{1}, X\right\rangle+\left\langle D_{1}, Y\right\rangle+\left\langle\bar{\gamma}_{1}, b_{1}-\mathcal{A}_{1} X-\mathcal{B}_{1} Y\right\rangle+\left\langle\bar{\gamma}_{2}, b_{2}-\mathcal{A}_{2} X-\mathcal{B}_{2} Y\right\rangle \\
& +\left\langle\bar{\gamma}_{3}, \mathcal{B}_{2}^{*} \lambda-D_{2}\right\rangle+\bar{\mu}_{1}\left[\left\langle\mathcal{A}_{2} X+\mathcal{B}_{2} Y-b_{2}, \lambda\right\rangle\right]+\bar{\mu}_{2}\left[\left\langle D_{2}-\mathcal{B}_{2}^{*} \lambda, Y\right\rangle\right] .
\end{aligned}
$$

If 


$$
\left\{\begin{array}{l}
b_{1}-\mathcal{A}_{1} X-\mathcal{B}_{1} Y \leq 0 \\
b_{2}-\mathcal{A}_{2} X-\mathcal{B}_{2} Y \leq 0 \\
\mathcal{B}_{2}^{*} \lambda-D_{2} \preceq 0 \\
\left\langle\mathcal{A}_{2} X+\mathcal{B}_{2} Y-b_{2}, \lambda\right\rangle=0 \\
\left\langle D_{2}-\mathcal{B}_{2}^{*} \lambda, Y\right\rangle=0
\end{array},\right.
$$

then $\left\langle C_{1}, \bar{X}\right\rangle+\left\langle D_{1}, \bar{Y}\right\rangle \leq\left\langle C_{1}, X\right\rangle+\left\langle D_{1}, Y\right\rangle$, which implies $(\bar{X}, \bar{Y}, \bar{\lambda})$ is the minimum point of $(P)$.

It follows from (4.3) that

$$
\begin{aligned}
\left\langle C_{1}, \bar{X}\right\rangle+\left\langle D_{1}, \bar{Y}\right\rangle \leq & \left\langle C_{1}, X\right\rangle+\left\langle D_{1}, Y\right\rangle+\left\langle\bar{\gamma}_{1}, b_{1}-\mathcal{A}_{1} X-\mathcal{B}_{1} Y\right\rangle+\left\langle\bar{\gamma}_{2}, b_{2}-\mathcal{A}_{2} X-\mathcal{B}_{2} Y\right\rangle \\
& +\left\langle\bar{\gamma}_{3}, \mathcal{B}_{2}^{*} \lambda-D_{2}\right\rangle+\bar{\mu}_{1}\left[\left\langle\mathcal{A}_{2} X+\mathcal{B}_{2} Y-b_{2}, \lambda\right\rangle\right]+\bar{\mu}_{2}\left[\left\langle D_{2}-\mathcal{B}_{2}^{*} \lambda, Y\right\rangle\right] \\
= & \varphi(\bar{\gamma}, \bar{\mu}) .
\end{aligned}
$$

As $\gamma_{1}, \gamma_{2} \geq 0, \gamma_{3} \succeq 0$, we prove that $(\bar{\gamma}, \bar{\mu})$ is the maximum point of $(D)$.

\section{Conclusions}

This paper mainly demonstrates the Lagrange duality of matrix linear bilevel programming, including the Lagrange dual function, weak duality and strong duality. The relationship between saddle point and optimal point is also discussed. When both the problem $(P)$ and $(D)$ have optimal solutions, the corresponding Lagrange function does not necessarily have a saddle point. But if the dual gap at the solution point is zero, the optimal condition is the saddle point condition. When both the problem $(P)$ and $(D)$ have strictly feasible solutions, the primal problem and the dual problem are solvable and the dual gap is zero. The saddle point of Lagrange function and the optimality condition are completely equivalent. Other dual forms such as conjugate dual will be studied in the future. The duality of nonlinear bilevel programming problems even if the optimality theory in matrix space will be our next objective.

\section{Funding}

The paper is supported by the National Natural Science Foundation of China under project No.61573228 and No.11501080.

\section{References}

[1] Bracken, J. and McGill, J. T. (1973). Mathematical programs with optimization problems in the constraints [J]. Operations Research, 1973, 21: 37-44.

[2] Wang, Q. P. and Ding, M. (2016). Bilevel programming model of traffic microcirculation optimization for historic district [J]. Journal of Traffic and Transportation Engineering, 2016, 16(3): 125-132.

[3] Ren, A. H. (2014). Research and application of several kinds of complex bilevel programming problems [D]. Xi'an, Xidian University, 2014.

[4] Colson, B., Marcotte, P., and Savard, G. (2007). An overview of bilevel programming [J]. Annals of Operations Research, 2007, 153: 235-256.

[5] Dempe, S. (2002). Foundations of Bilevel Programming, Nonconvex optimization and its applications [M]. Dordrecht: Kluwer Acadermic, 2002.

[6] Luo, Z. Q., Pang, J. S., and Ralph, D. (1996). Mathematical programs with equilibrium constraints [M]. Cambridge: Cambridge University Press.

[7] Ye, J. J. (2005). Necessary and sufficient optimality conditions for mathematical programs with equilibrium constraints [J]. Journal of Mathematical Analysis and Applications, 307(2005): 305-369.

[8] Huang, Z. H., Lin, G. H., and Xiu, N. H. (2014). Several developments of variational inequalities and complementarity problems, bilevel programming and MPEC [J]. Operations Research Transactions, 2014, 18(01): 113-133.

[9] Su, J. and Ma, J. H. (2003). Surrogate dual of bilevel linear programming [J]. Journal of Shandong University, 2003, 38(5): 45-51.

[10] Xie, Z. X. and Xie, T. J. (2008). Duality programming for solution-type linear bilevel programming [J]. Journal of Beijing Technology and Business University (Natural Science Edition), 2008, 26(2): 81-84. 